\title{
Initiation characteristics of wedge-induced oblique detonation waves in a stoichiometric hydrogen-air mixture
}

\author{
Honghui Teng ${ }^{\mathrm{a}, *}$, Hoi Dick Ng ${ }^{\mathrm{b}}$, Zonglin Jiang ${ }^{\mathrm{a}}$ \\ ${ }^{a}$ State Key Laboratory of High Temperature Gas Dynamics, Institute of Mechanics, Chinese Academy of Sciences, Beijing \\ 100190, China \\ ${ }^{\mathrm{b}}$ Department of Mechanical and Industrial Engineering, Concordia University, Montréal H3G 1 M8, Canada
}

Received 30 November 2015; accepted 27 September 2016

Available online 10 October 2016

\begin{abstract}
The initiation features of two-dimensional, oblique detonations from a wedge in a stoichiometric hydrogen-air mixture are investigated via numerical simulations using the reactive Euler equations with detailed chemistry. A parametric study is performed to analyze the effect of inflow pressure $P_{0}$, and Mach number $M_{0}$ on the initiation structure and length. The present numerical results demonstrate that the two transition patterns, i.e., an abrupt transition from a multi-wave point connecting the oblique shock and the detonation surface and a smooth transition via a curved shock, depend strongly on the inflow Mach number, while the inflow pressure is found to have little effect on the oblique shock-to-detonation transition type. The present results also reveal a slightly more complex structure of abrupt transition type in the case of $M_{0}=7.0$, consisting of various chemical and gasdynamic processes in the shocked gas mixtures. The present results show quantitatively that the initiation length decreases with increasing $M_{0}$, primarily due to the increase of post-shock temperature. Furthermore, the effect of $M_{0}$ on initiation length is independent of $P_{0}$, but given the same $M_{0}$, the initiation length is found to be inversely proportional to $P_{0}$. Theoretical analysis based on the constant volume combustion (CVC) theory is also performed, and the results are close to the numerical simulations in the case of high $M_{0}$ regardless of $P_{0}$, demonstrating that the post-oblique-shock condition, i.e., post-shock temperature, is the key parameter affecting the initiation. At decreasing $M_{0}$, the CVC theory breaks down, suggesting a switch from chemical kinetics-controlled to a wave-controlled gasdynamic process. For high inflow pressure $P_{0}$ at decreasing $M_{0}$, the CVC theoretical estimations depart from numerical results faster than those of low $P_{0}$, due to the presence of the non-monotonic effects of chemical kinetic limits in hydrogen oxidation at high pressure.
\end{abstract}

(C) 2016 The Combustion Institute. Published by Elsevier Inc. All rights reserved.

Keywords: Oblique detonation; Formation structure; Hydrogen; Shock wave; Detailed chemistry

\footnotetext{
* Corresponding author. Fax: +86 1082543995.

E-mail addresses: hhteng@imech.ac.cn, honghuiteng@gmail.com (H. Teng).
} 


\section{Introduction}

In recent years, the idea of using oblique detonation waves (ODWs) for high efficiency propulsion systems has generated great interest for the development of air-breathing hypersonic aircraft [1-3]. This hypersonic propulsion concept not only has the advantages of the scramjet, but also achieves high thermal cycle efficiency through the detonation mode of combustion [3]. However, for proper design and operation of ODW engines, one must predict the necessary conditions and understand the mechanism behind ODW initiation from a wedge.

Classical theory on ODWs simplifies the structure into an oblique shock with a post-shock energy release zone attached to the wedge $[4,5]$. From shock polar analysis, the relationship between different flow parameters and oblique shock/detonation properties can be determined, e.g., [6-8]. The formation of oblique detonations was first analyzed numerically by $\mathrm{Li}$ et al. [9], who observed a structure composed of a nonreactive oblique shock, an induction region followed by a reaction region, along the wall, and the oblique detonation surface. This abrupt, steady, oblique shock-to-detonation transition by a multi-wave point has been confirmed experimentally and considered as the standard structure used widely in subsequent studies [10-12].

Under realistic conditions, multi-dimensional, gaseous detonation waves are inherently unstable [13]. The formation of unstable cellular structures on the oblique detonation surface is revealed numerically downstream of the kink-like, oblique shock-to-detonation transition [14-20], equivalent to a normal cellular detonation. Even with a high degree of overdrive, the oblique detonation under normal conditions becomes unstable, but with a low instability growth rate [17].

Recent investigations found that both abrupt and smooth transition structures exist [21-25]. Unlike the widely-studied abrupt mechanism, the smooth transition originates from a curved shock instead of the multi-wave point. Simulations indicate that the type of transition processes depend on chemical and aerodynamic parameters. In particular, the inflow Mach number toward the wedge and chemical activation energy are critical for the formation mechanism of an ODW. The recent numerical simulations along with shock-polar analysis using a two-step, chemical reaction model by Liu et al. [26] illustrate clearly the effect of Mach number on the change of formation mechanism of the ODW structures.

In this work, numerical simulations are conducted to further investigate the initiation characteristics of an ODW from a wedge. As shown from [22-24], both aerodynamic and mixture thermodynamic properties greatly influence the detonation initiation. As such, a parametric study is

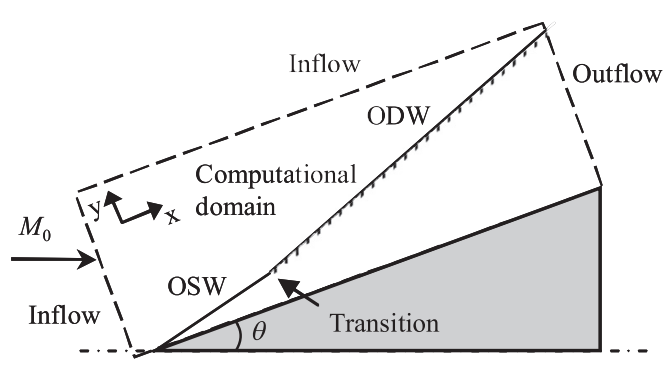

Fig. 1. Schematic of a typical oblique detonation wave.

performed using two bifurcation parameters: the incident Mach number $M_{0}$, and inflow pressure $P_{0}$. Most previous studies $[9,15-20,26]$ used simplified chemical reaction models, mainly the one- or two-step irreversible Arrhenius-type, temperaturedependent kinetic model. The influence of $M_{0}$ is analyzed in almost all of these investigations, but the effect of inflow pressure $P_{0}$ is not typically discussed due to the use of simplified chemical kinetics. To address this drawback, ODWs in a stoichiometric hydrogen-air mixture are investigated here with detailed chemistry. The effect of both $M_{0}$ and $P_{0}$ on the initiation characteristics are analyzed, as in $[17,20]$, by looking at the variation of a characteristic length scale describing the initiation process.

\section{Computational details}

Figure 1 shows schematically a typical ODW induced by a wedge in a combustible gas mixture. The presence of a wedge in supersonic inflow induces first an oblique shock wave (OSW). For a high inflow Mach number causing a high post-shock temperature behind the OSW, an exothermic reaction begins, leading to the formation of an ODW. For the computation, the coordinate is rotated to the direction along the wedge surface. Hence, the Cartesian grid in the rectangular domain enclosed by the dashed line is aligned with the wedge surface. Similar to previous numerical studies [21,25], the analysis is based on the two-dimensional, multi-species Euler equations given by:

$$
\frac{\partial \mathbf{U}}{\partial t}+\frac{\partial \mathbf{F}}{\partial x}+\frac{\partial \mathbf{G}}{\partial y}=\mathbf{S}
$$

where:

$$
\mathbf{U}=\left\{\begin{array}{c}
\rho_{1} \\
\vdots \\
\rho_{n} \\
\rho u \\
\rho v \\
e
\end{array}\right\}, \mathbf{F}=\left\{\begin{array}{c}
\rho_{1} u \\
\vdots \\
\rho_{n} u \\
\rho u^{2}+p \\
\rho u v \\
(e+p) u
\end{array}\right\}, \mathbf{G}=\left\{\begin{array}{c}
\rho_{1} v \\
\vdots \\
\rho_{n} v \\
\rho u v \\
\rho v^{2}+p \\
(e+p) v
\end{array}\right\},
$$




$$
\mathbf{S}=\left\{\begin{array}{c}
\omega_{1} \\
\vdots \\
\omega_{n} \\
0 \\
0 \\
0
\end{array}\right\}
$$

The total density and energy are calculated by:

$\rho=\sum_{i=1}^{n} \rho_{i}, e=\rho h-p+\frac{1}{2} \rho\left(u^{2}+v^{2}\right)$,

where specific enthalpy can be written as $h=$ $\sum_{i=1}^{n} \rho_{i} h_{i} / \rho$ with $h_{i}$ obtained from the thermodynamic data of each specie. The equation of state is

$p=\sum_{i=1}^{n} \rho_{i} \frac{R_{0}}{w_{i}} T$

where $w_{i}$ is the molecular weight, $T$ is the gas temperature, and $\omega_{i}$ is the species' specific mass production rate, dictated by the chemical reaction model. In this study, a hydrogen mechanism for high-pressure combustion [27] is considered. This mechanism involves 27 reversible, elementary reactions among the 8 species $\mathrm{H}_{2}, \mathrm{O}_{2}, \mathrm{H}_{2} \mathrm{O}, \mathrm{H}, \mathrm{O}$, $\mathrm{OH}, \mathrm{HO}_{2}$, and $\mathrm{H}_{2} \mathrm{O}_{2}$, with 5 inert species, $\mathrm{N}_{2}, \mathrm{Ar}$, $\mathrm{He}, \mathrm{CO}$, and $\mathrm{CO}_{2}$. Thermodynamic properties of these species are evaluated from the 9-coefficient NASA polynomial representation [28]. Although the details of chemical kinetics are well known to play a major role in modeling detonation cell structures [29], this issue is less vital to this study. It is not the objective here to assess whether a reaction model can reproduce cell sizes or any resolved, experimental data. Instead, a well-validated, chemical kinetic model is chosen to examine the effect of Mach number and initial pressure, the initiation mechanism, and to compare CFD results with a spatially homogeneous constant-volume combustion model [25] to shed light on the ODW formation. The chemical kinetic model used here is also one of the most recent, detailed, hydrogen mechanisms suitable for high-pressure combustion. Validation tests, relevant to detonation conditions and ODW initiation structures are provided in [27], including high-pressure ignition delay times, species evolution in shock tubes and well-stirred reactors, and explosion limits. The chemical kinetic mechanism by Burke et al. [27] is updated from [30], shown to accurately estimate the ZND reaction length for cell size prediction [31]. Although the recent work by Taylor et al. [29] found this model cannot exactly match experimental detonation cells, this should not affect the major ODW initiation structure analyzed here. Nevertheless, there is no doubt that if the detonation cell structure is of particular concern, as pointed out in [29], other processes should be considered in future works to reproduce cell measurements, such as chemistry mod-
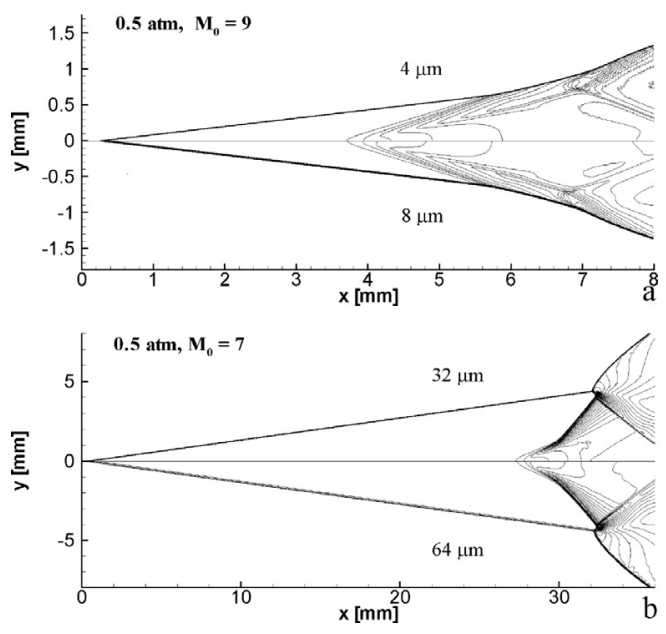

Fig. 2. Results from the grid resolution study.

els taking into account vibrational non-equilibrium [32]. The reduction, in Euler simulations, of numerical diffusion with higher numerical resolution must also be considered. To reproduce detonation cells and capture different reaction scales, physical diffusion is likely as important as fast reaction $[33,34]$, especially for highly unstable cellular detonations [35]. Solving the Navier-Stokes equations with physical diffusion and viscosity are thus necessary to achieve true numerical convergence.

The governing equations are discretized on Cartesian uniform grids and solved with the dispersion-controlled dissipative (DCD) scheme [36] with Strang's splitting. To overcome the stiffness problem, a sufficient number of sub-reaction steps are involved to assure overall accuracy [37]. A stoichiometric hydrogen-air mixture with $\mathrm{H}_{2}: \mathrm{O}_{2}: \mathrm{N}_{2}=2: 1: 3.76$ is used. The slip reflecting boundary condition is used on the wedge surface and the other boundaries are interpolated assuming zero first-order derivatives of all flow parameters. On the lower computational boundary, the wedge starts from $x=0.256 \mathrm{~mm}$ for all cases. Unless specified, a $25^{\circ}$ wedge angle is used. Because of the multi-scale nature of the phenomena, both the computational domain and mesh scale are adjusted. The numerical grid resolution used for different cases varies from a coarsest mesh of $64 \mu \mathrm{m}$ to the finest mesh of $2 \mu \mathrm{m}$. The results are also examined to ensure the ODW initiation structure is unaffected by mesh properties. For instance, a sample examining the effect of mesh size is shown in Figs. 2 and 3. The difference between the simulated structure using the chosen mesh size and a case with twice the resolution is found to be negligible. Although a discrepancy appears in the $\mathrm{OH}$ concentration near the oblique detonation surface which can be due to the unsteadiness of the ODW structure as well, for the purpose of this work, the 

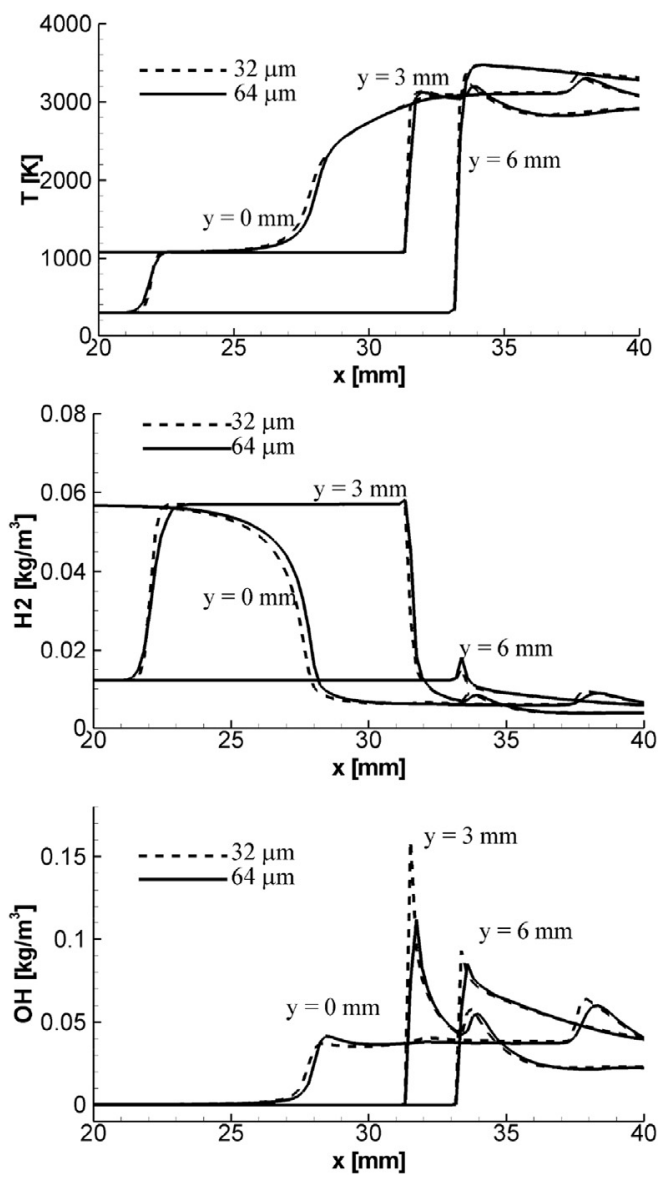

Fig. 3. Results from the grid resolution study for $P_{0}=0.5 \mathrm{~atm}$ and $M_{0}=7$ showing the temperature and species profiles along different $y$-line locations.

chosen grid size provides converged, global, formation structures, sufficient to guarantee the reliability of the conclusion. Nevertheless, a higher resolution is certainly desirable to resolve the small-scale details within the wave structure, outside the scope of this study.

\section{Results and discussion}

The pressure and temperature fields obtained are shown in Fig. 4, with $P_{0}=1.0 \mathrm{~atm}$ and $M_{0}$ varied from 7.0 to 10.0. In Fig. 4, the scale extent is adjusted to cover all initiation features. The combustion process in the shocked gas changes. For $M_{0}=10.0$, a significant temperature increase near the wedge can be observed before $x=1.5 \mathrm{~mm}$, and a curved oblique shock/detonation surface appears, as shown in Fig. 4a. By decreasing $M_{0}$ to 9.0, similar features are observed, but the initiation position moves further downstream. For $M_{0}=8.0$, a multi- wave point forms where the oblique shock-todetonation transition is achieved abruptly, rather than gradually through a curved shock. The case of $M_{0}=8.0$ recovers the typical structure, with a set of pressure waves from the exothermic reactions in the shocked gas, described in the literature.

In the case of $M_{0}=7.0$ (Fig. $4 \mathrm{~d}$ ), the wave structure becomes more complex. The reaction surface in the shocked gas is composed of two sections with different oblique angles: the lower one close to the wedge has a small oblique angle, while the upper one connects with the multi-wave point and has a greater oblique angle. The lower section has gradually-increasing compression waves attached, similar to Fig. 4c. The upper section is coupled with a strong oblique shock, demonstrating the appearance of a secondary oblique detonation in the shocked gas.

Oblique detonations with $P_{0}=0.5$ and 0.2 atm are simulated to look at the effect of inflow pressure (Figs. 5 and 6). These results show that $P_{0}$ has little effect on the initiation structure, i.e., the curved shock appears in the case of high $M_{0}$ and the multi-wave point appears in the case of low $M_{0}$. For $M_{0}=7.0$, the complex combination of reaction waves and oblique detonation appears for all $P_{0}$. Because the structures are all found to be similar, flow fields obtained for $M_{0}=9.0$ and 8.0 are not displayed here. The position of the ODW initiation, although, is influenced by $P_{0}$. At first, it appears that given a value of $M_{0}$, the initiation position moves further and further downstream with decreasing $P_{0}$, despite the structures remaining similar. This can be explained from the fact that a lower $P_{0}$ corresponds to a lower mixture density, increasing the initiation time. However, such monotonic variation between the initiation time and $P_{0}$ is not always the case, particularly for low $M_{0}$, due to chemical kinetic characteristics of hydrogen oxidation. The influence of $P_{0}$ on the initiation length will be analyzed in detail later in the analysis.

Although the structures shown in Fig. 4a-c have been studied widely, the one appearing in Fig. 4d, characterized by two distinct reaction surfaces in the shocked gas, has not been fully described. Similar structures have also been presented recently by Liu et al. [26] using an ideal model with two-step chemical kinetics. Figure 7 shows the pressure and temperature along different lines parallel to the $x$ axis, i.e., the wedge surface, obtained from the case of $P_{0}=0.5 \mathrm{~atm}$ and $M_{0}=7.0$, to illustrate different combustion characteristics inside this complex structure. Along the black line corresponding to the wedge, mild increases in pressure and temperature can be observed, and a weak compression wave begins to form. Along the green line, corresponding to the triggered exothermic reaction in the shocked gas, a strong compression wave is generated, which further promotes heat release. Along the red line, corresponding to the secondary oblique detonation in the shocked gas, one large pressure peak can 

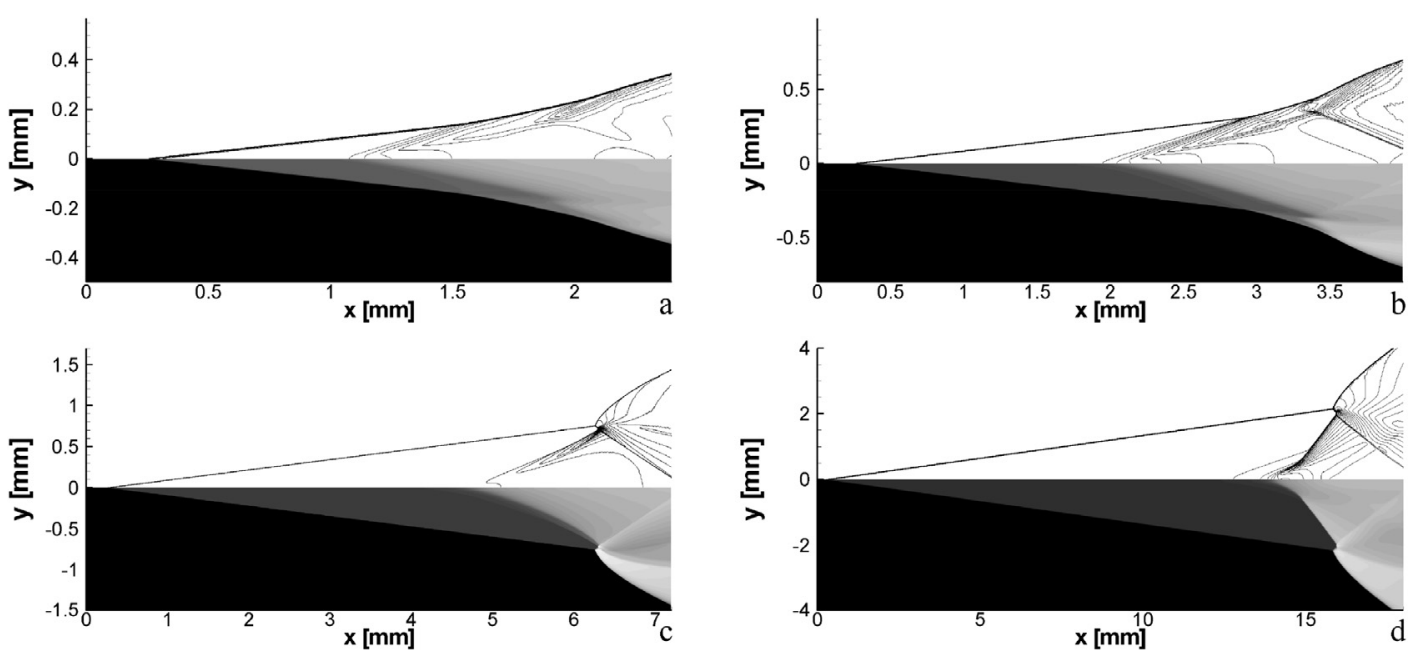

Fig. 4. Pressure (upper) and temperature (lower) fields with $P_{0}=1.0 \mathrm{~atm}$ and $M_{0}=$ (a) 10.0, (b) 9.0, (c) 8.0, and (d) 7.0.
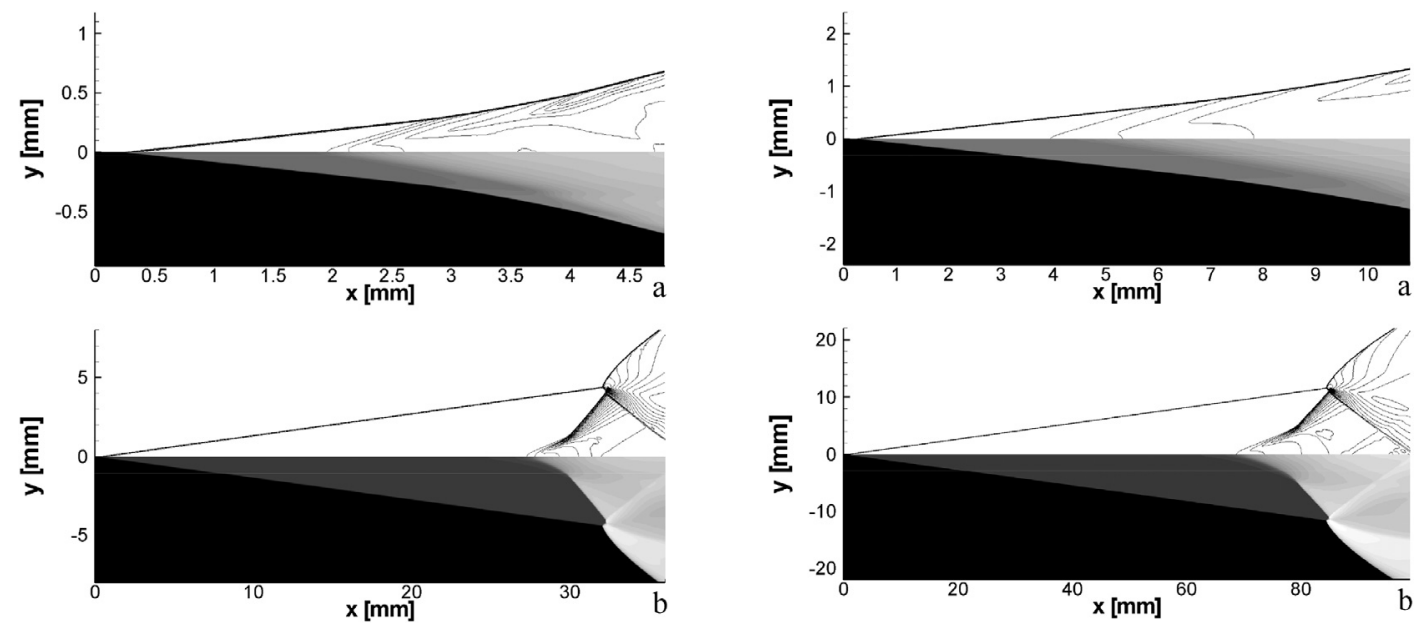

Fig. 5. Pressure (upper) and temperature (lower) fields with $P_{0}=0.5$ atm and $M_{0}=$ (a) 10.0 and (b) 7.0 .

be observed. Compared with the blue curve, corresponding to the main oblique detonation wave, the oblique detonation in the shocked gas has a higher pressure peak, but lower temperature rise.

Although these results demonstrate that the initiation structure is mainly influenced by $M_{0}$, the initiation positions are also affected by $P_{0}$. To further quantify the effect of these two parameters, a characteristic initiation length, $L_{\mathrm{c}}$ is considered [25]. $L_{\mathrm{c}}$ is defined along the flow stream direction, parallel with the $x$-axis. It starts from the oblique shock and terminates at the end of the induction zone, i.e., the location at which the temperature increases over $10 \%$. The lengths are different depending on the distance from the wedge, and thus, the charac-

Fig. 6. Pressure (upper) and temperature (lower) fields with $P_{0}=0.2 \mathrm{~atm}$ and $M_{0}=$ (a) 10.0 and (b) 7.0.

teristic length of initiation is given by the maximum value for each case of $\left(M_{0}, P_{0}\right)$, always located on the wedge surface.

Figure 8 shows the initiation length as a function of $M_{0}$ with $P_{0}=0.2,0.5$, and $1.0 \mathrm{~atm}$. For a given $P_{0}, L_{\mathrm{c}}$ decreases when $M_{0}$ increases. The decrease in $L_{\mathrm{c}}$ can be explained by the increase in post-shock temperature. In addition, one can see the three dashed lines are almost parallel with each other, demonstrating the effect of $M_{0}$ on initiation length is independent of $P_{0}$. Given the same $M_{0}$, numerical simulations show $L_{\mathrm{c}}$ decreases when $P_{0}$ increases. Moreover, $L_{\mathrm{c}}$ is almost inversely proportional to $P_{0}$. Table 1 shows the estimation errors for the initiation length in the case of 

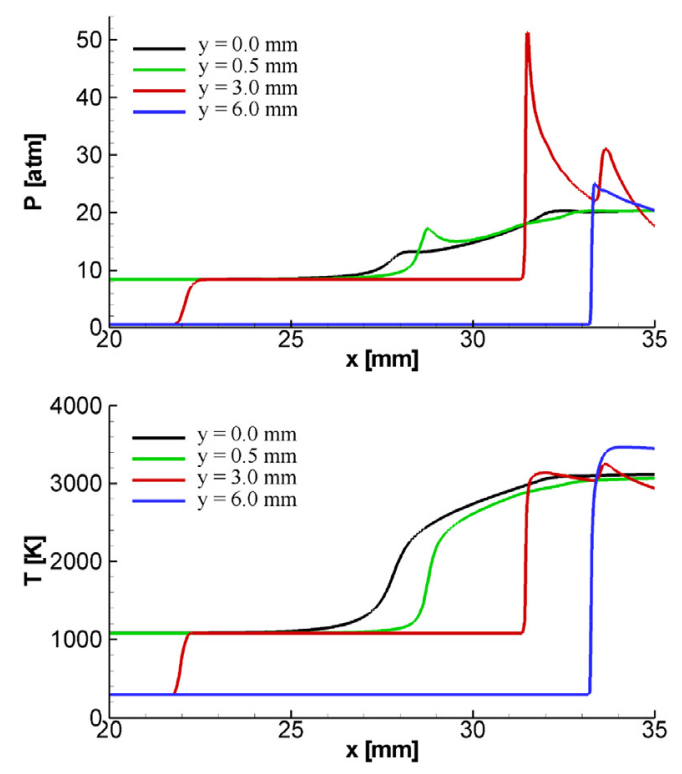

Fig. 7. Pressure (upper) and temperature (lower) profiles along the lines parallel with the $x$-axis with $P_{0}=0.5 \mathrm{~atm}$ and $M_{0}=7.0$.

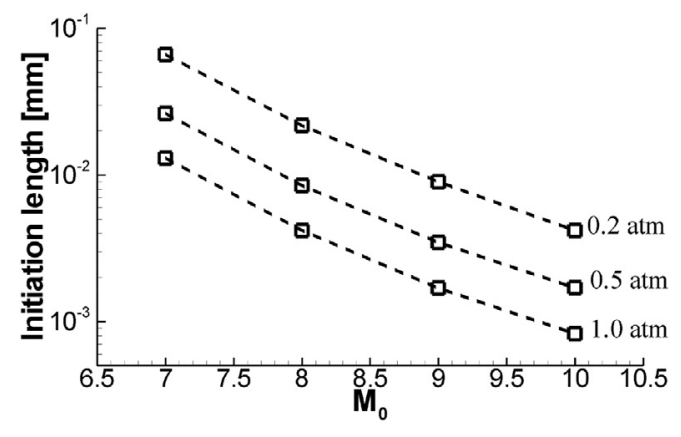

Fig. 8. The dependence of the initiation length on $M_{0}$ with $P_{0}=0.2,0.5$, and 1.0 atm.

\section{Table 1}

Initiation length errors calculated from the case of $P_{0}=0.5 \mathrm{~atm}$.

\begin{tabular}{rcc}
\hline \multicolumn{1}{c}{$M_{0}$} & $P_{0}=1.0 \mathrm{~atm}(\%)$ & $P_{0}=0.2 \mathrm{~atm}(\%)$ \\
\hline 10.0 & 0.30 & 0.70 \\
9.0 & 1.63 & 0.72 \\
8.0 & 5.02 & -0.41 \\
7.0 & 10.52 & -9.25 \\
\hline
\end{tabular}

$P_{0}=0.2$ and 1.0 atm, calculated using an inversely proportional relationship based on the simulation value of $P_{0}=0.5 \mathrm{~atm}$. Overall, the error is bounded within about $10 \%$, and is negligible, particularly at high $M_{0}$. The latter can be attributed to the simple ODW initiation structure observed for high $M_{0}$. For low $M_{0}$, the transition and initiation structures

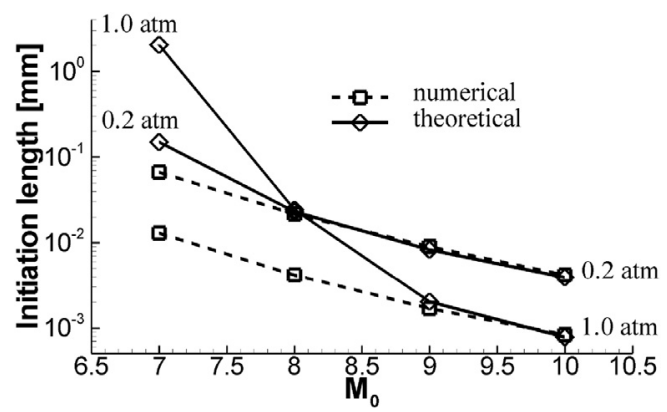

Fig. 9. Numerical and theoretical initiation length dependence on $M_{0}$ with $P_{0}=0.2$ and $1.0 \mathrm{~atm}$.

are more complex, as shown in Figs. 4-6, causing the discrepancies. Nevertheless, the inverse rule gives a good zero-order characterization of $L_{\mathrm{c}}\left(P_{0}\right)$.

Following [25], a theoretical approach based on constant volume combustion (CVC) theory is used to estimate $L_{\mathrm{c}}$ with post-oblique-shock conditions. It assumes a flow structure where, near the wedge, the mixture is completely burned, and pressure build-up and the formation of pressure waves are weak. The initiation is assumed to be kineticallycontrolled. In this work, all calculations are performed using the CHEMKIN package [38]. First, the post-oblique-shock specie densities and temperature are used to simulate CVC to obtain the reaction time required to attain a mixture temperature with a $10 \%$ increase from its post-shock value. The initiation length $L_{\mathrm{c}}$ is then calculated by multiplying the time with the post-oblique-shock particle velocity. Despite a simple formulation, this analysis provides a predictive approach for the general structure of oblique detonations [25].

Figure 9 shows the results of $L_{\mathrm{c}}$ variations with different $M_{0}$ for $P_{0}=0.2$ and $1.0 \mathrm{~atm}$ obtained from both the numerical simulations and CVC estimations. For $P_{0}=0.2 \mathrm{~atm}$, both results are in good agreement (with a noticeable deviation only at low $M_{0}$ ). In contrast, a greater discrepancy can be seen for $P_{0}=1.0 \mathrm{~atm}$, starting at $M_{0}=8.0$. It appears more difficult to predict the initiation length in the case of high $P_{0}$ using this simplified chemical kinetic approach. Overall, the theoretical and numerical results are close for high $M_{0}$ regardless of $P_{0}$. When $M_{0}$ decreases, the present theory deviates faster in the case of high $P_{0}$.

In Fig. 9, the theoretical results are seen to deviate for $M_{0} \leq 8.0$. To shed light on this observation, the theoretical initiation length is shown in Fig. 10 as a function of both $M_{0}$ and $P_{0}$. Note that given $M_{0}$, the post-shock temperature and velocity are fixed, and the pressure and density vary with $P_{0}$. From Fig. 10, we see that the initiation length decreases monotonically with increasing $P_{0}$ in the case of high $M_{0}$, i.e., $M_{0}=10.0$ and 9.0 . 


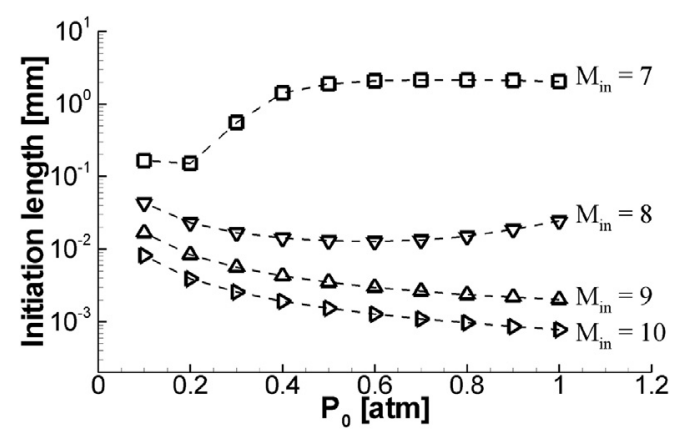

Fig. 10. The dependence of initiation length on $P_{0}$ from the theoretical prediction.

However, for $M_{0}=8.0$, a minimum initiation length appears near $P_{0}=0.6 \mathrm{~atm}$ and the variation reverses above $P_{0}=0.6 \mathrm{~atm}$ where the initiation length increases with increasing $P_{0}$. This nonmonotonic variation is more apparent in the case of $M_{0}=7.0$. A similar phenomenon is also observed in $[31,39]$, which examined the chemical kinetics behavior and assessment of detonation hazards in high-pressure hydrogen-air mixture from ZND analysis. Due to the explosion characteristics of hydrogen, at elevated pressure the second explosion limit effect plays a significant role leading to slow branching reactions. As a result, the overall reaction kinetic rate is reduced resulting in a longer induction time of the combustion process. This behavior agrees with the results in Fig. 9 where an exponential increase appears in the theoretical initiation length from the CVC calculation at $P_{0}=1 \mathrm{~atm}$ below $M_{0}=8$. Since the results from the numerical simulations do not follow this exponential increase, it suggests that the initiation process is no longer controlled solely by the initiation kinetics. Thus, other mechanisms should come into play affecting the initiation. As shown in Fig. 4, a switch in the transition type from a smooth curve shock to an abrupt multi-wave point appears at $M_{0}=8$ for $P_{0}=1 \mathrm{~atm}$. The onset of deviation between the theoretical estimation and the numerical results provides an indication of the transition type, and the initiation changes from a kinetically-controlled process behind the oblique shock to a wave-controlled, gasdynamic initiation phenomenon. The presence of stronger compression waves and the secondary oblique detonation in the shocked gas prevent $L_{\mathrm{c}}$ from increasing exponentially, reducing the unfavorable effect of hydrogen chemical kinetic limits.

The present results agree with our recent study; in the case of low pressure and high temperature inflow - conditions derived from the high altitude flight conditions $-L_{\mathrm{c}}$ can be well predicted by CVC theory [25]. In this study, CVC theoretical results are found to agree for high $M_{0}$ (i.e., $M_{0}=9$ and 10) regardless of $P_{0}$, where the transition is de- scribed by a structure with a smooth, curved shock. This further demonstrates that, at these conditions, oblique shock-induced reaction or the post-shock temperature is the key parameter controlling the initiation. However, at decreasing $M_{0}$, theoretical results at high $P_{0}$ diverge faster than those at low $P_{0}$ due to the non-monotonic effects of hydrogen oxidation with kinetic-controlled limits. The resulting, complex, gasdynamic structure for low $M_{0}$ leads to the breakdown of CVC prediction.

\section{Conclusion}

Two-dimensional, oblique detonations induced by a wedge were simulated to study the initiation structure and length with varying inflow Mach number $M_{0}$ and pressure $P_{0}$ in a stoichiometric hydrogen-air mixture. Numerical results illustrate that oblique detonation initiation has two transition patterns: an abrupt transition from a multiwave point connecting the oblique shock and the detonation surface, and a smooth transition via a curved shock. The transition type is found to depend strongly on $M_{0} . P_{0}$ is varied between 1.0 and $0.2 \mathrm{~atm}$, and the simulation results show that it has little effect on the initiation structure. A complex structure is revealed for $M_{0}=7.0$ where the flow field consists of various chemical-gasdynamic processes in the shocked gas mixtures. Analysis of the characteristic initiation length, defined along the flow stream direction, from the oblique shock and terminating at the end of the induction zone, shows it decreases with increasing $M_{0}$ primarily due to the increase in post-shock temperature. The effect of $M_{0}$ on the initiation length is independent of $P_{0}$. The present numerical results also show that given $M_{0}, L_{\mathrm{c}} \propto 1 / P_{0}$.

Chemical kinetic calculations based on CVC theory were performed to estimate the initiation length and compared with numerical simulations. The CVC results are shown to be close to the numerical ones for high $M_{0}$ regardless of $P_{0}$, demonstrating that the post-oblique-shock condition, i.e., post-shock temperature, is the key parameter and the initiation is kinetically-controlled. At lower $M_{0}$, theoretical results for high $P_{0}$ diverge faster than for low $P_{0}$ due to the non-monotonic behavior of the induction kinetics at elevated pressure from the hydrogen explosion characteristics of hydrogen. The complex, gasdynamic structure observed at low $M_{0}$ eventually causes the breakdown of CVC predictions. The deviation between the theoretical estimation and numerical results provides an indication of the transition mechanism from a kineticallycontrolled process in the gas shocked by the oblique shock to a wave-controlled gasdynamic initiation phenomenon. In the latter, the presence of stronger compression waves and the secondary oblique detonation in the shocked gas keep $L_{\mathrm{c}}$ from increasing 
exponentially and compensate the reverse effect of chemical kinetic limits in hydrogen.

\section{Acknowledgment}

This work is supported by NSFC, nos. 11372333 and 51376165.

\section{References}

[1] K. Kailasanath, AIAA J. 41 (2003) 145-159.

[2] A.J. Higgins, J. Propul. Power 22 (2006) 1170-1187.

[3] P. Wolanski, Proc. Combust. Inst. 34 (1) (2013) 125-158.

[4] D.T. Pratt, J.W. Humphrey, D.E. Glenn, J. Propul. Power 7 (5) (1991) 837-845.

[5] J.M. Powers, K.A. Gonthier, Phys. Fluids A 4 (8) (1992) 2082-2089

[6] J.M. Powers, D.S. Stewart, AIAA J. 30 (3) (1992) 726-736.

[7] S.A. Ashford, G. Emanuel, Shock Waves 3 (4) (1994) 327-329.

[8] C.I. Morris, M.R. Kamel, R.K. Hanson, Proc. Combust. Inst. 27 (1998) 2157-2164.

[9] C. Li, K. Kailasanath, E.S. Oran, Phys. Fluids 4 (1994) 1600-1611.

[10] C. Viguier, L. Figueira da Silva, D. Desbordes, B. Deshaies, Proc. Combust. Inst. 26 (1996) 3023-3031.

[11] J.P. Sislian, H. Schirmer, R. Dudebout, J. Schumacher, J. Propul. Power 17 (3) (2001) 599-604.

[12] G. Fusina, J.P. Sislian, B. Parent, AIAA J. 43 (7) (2005) 1591-1604

[13] H.D. Ng, F. Zhang, Shock Waves Science and Technology Library, Vol. 6, in: F. Zhang (Ed.), Springer, Berlin Heidelberg, 2012 Chap. 3.

[14] M.J. Grismer, J.M. Powers, Shock Waves 6 (1996) 147-156.

[15] M.V. Papalexandris, Combust. Flame 120 (2000) 526-538.

[16] J.Y. Choi, D.W. Kim, I.S. Jeung, F. Ma, V. Yang, Proc. Combust. Inst. 31 (2007) 2473-2480.

[17] H.H. Teng, Z.L. Jiang, H.D. Ng, J. Fluid Mech. 744 (2014) 111-128.

[18] M.Y. Gui, B.C. Fan, G. Dong, Acta Mech. Sin. 27 (2011) 922-928.
[19] J. Verreault, A.J. Higgins, R.A. Stowe, Proc. Combust. Inst. 34 (2013) 1913-1920.

[20] H.H. Teng, H.D. Ng, K. Li, C.T. Luo, Z.L. Jiang, Combust. Flame 162 (2015) 470-477.

[21] L. Figueira da Silva, B. Deshaies, Combust. Flame 121 (2000) 152-166.

[22] A.F. Wang, W. Zhao, Z.L. Jiang, Acta Mech. Sin. 27 (2011) 311-619

[23] H.H. Teng, Z.L. Jiang, J. Fluid Mech. 713 (2012) 659-669.

[24] J.Y. Choi, E.J.R. Shin, I.S. Jeung, Proc. Combust. Inst. 32 (2009) 2387-2396.

[25] T. Wang, Y. Zhang, H.H. Teng, Z.L. Jiang, H.D. Ng, Phys. Fluids 27 (2015) 096101.

[26] Y. Liu, Y.S. Liu, D. Wu, J.P. Wang, Shock Waves 26 (2) (2016) 161-168

[27] M.P. Burke, M. Chaos, Y. Ju, F.L. Dryer, S.J. Klippenstein, Int. J. Chem. Kinet. 44 (7) (2012) $444-474$.

[28] B.J. McBride, M.J. Zehe, S. Gordon, NASA Glenn Coefficients for Calculating Thermodynamic Properties of Individual Species, NASA/TP, 2002 Report No. 2002-211556.

[29] B.D. Taylor, D.A. Kessler, V.N. Gamezo, E.S. Oran, Proc. Combust. Inst. 34 (2013) 2009-2016.

[30] J. Li, Z. Zhao, A. Kazakov, F.L. Dryer, Int. J. Chem. Kinet. 36 (2004) 566-575.

[31] H.D. Ng, Y. Ju, J.H.S. Lee, Int. J. Hydrog. Energy 32 (2007) 93-99.

[32] L.S. Shi, H. Shen, P. Zhang, D.L. Zhang, C.Y. Wen, in: 2nd International Conference on high Temperature Gas Dynamics, June 23-25, Beijing, China, 2016, pp. 19-21.

[33] S. Paolucci, Z. Zikoski, D. Wirasaet, J. Comput. Phys. 272 (2014) 814-841.

[34] S. Paolucci, Z.J. Zikoski, T. Grenga, J. Comput. Phys. 272 (2014) 842-864.

[35] K. Mazaheri, Y. Mahmoudi, M.I. Radulescu, Combust. Flame 159 (6) (2012) 2138-2154.

[36] Z. Jiang, Acta Mech. Sin. 20 (1) (2004) 1-15.

[37] H.C. Yee, D.V. Kotov, W. Wang, C.W. Shu, J. Comp. Phys. 241 (2013) 266-291.

[38] R.J. Kee, F.M. Rupley, E. Meeks, J.A. Miller, CHEMKIN-III: A Fortran Chemical Kinetic Package for the Anaylsis of Gas-phase Chemical and Plasma Knietics, 1996 Tech. Rep. SAND96-8216, Sandia National Laboratories.

[39] T. Lu, C.K. Law, Y. Ju, J. Prop. Power 19 (5) (2003) 901-907. 